\title{
Novel agents in a young patient with osteosarcoma: A short review
}

\author{
Oskar Steinbrecher - Thomas Brodowicz · Wolfgang Lamm
}

Received: 14 April 2021 / Accepted: 16 June 2021 / Published online: 19 July 2021

(C) The Author(s) 2021

\begin{abstract}
Summary Osteosarcomas are rare malignant bone tumors, most frequently occurring in children as well as adolescents and young adults. Therapy of initially localized disease consists of neoadjuvant chemotherapy followed by surgical resection and adjuvant chemotherapy. Osteosarcomas often present relapses, most commonly lung metastases. Treatment of isolated lung metastasis most commonly includes surgical resection. The correct adjuvant treatment option is still under investigation. In this manuscript we describe the clinical course of an osteosarcoma patient and give a review of the literature regarding current standard treatment for localized as well as pulmonary metastatic disease.
\end{abstract}

Keywords Osteosarcoma · Pembrolizumab . Pazopanib · Regorafenib · Metastasectomy

\section{Introduction}

Osteosarcomas are rare malignancies, which most commonly occur in children as well as adolescents and young adults, aged between 15-25 years [1]. Initially localized osteosarcoma is generally treated with neoadjuvant chemotherapy followed by surgical resection and adjuvant chemotherapy [2, 3]. Osteosarcomas nevertheless frequently develop isolated pulmonary relapses, which are commonly treated with complete metastasectomy if possible, even in consecutively relapsed disease $[4,5]$. However, data on systemic treatment options after pulmonary re-

Dr. O. Steinbrecher · ao. Univ. Prof. Dr. T. Brodowicz • Assoc. Prof. PD Dr. W. Lamm ( $ه)$

Clinical Division of Oncology, Department of Medicine

I, Medical University of Vienna-General Hospital,

Waehringer Guertel 18-20, 1090 Vienna, Austria

wolfgang.lamm@meduniwien.ac.at lapse are limited; therefore, treatments are mostly assigned individually in a patient-tailored setting [2]. In this article, we describe a patient treated with various systemic treatments after metastasectomy and briefly summarize the current literature.

\section{Case report}

We describe the case of a 19-year-old woman who consulted her general practitioner in July 2013 due to acute and increasing pelvic pain. A computed tomography (CT) scan showed a suspicious mass of the left hemipelvis with a maximum diameter of $15.5 \mathrm{~cm}$ (Fig. 1). Subsequently, she was referred to the Vienna General Hospital, Medical University of Vienna, a specialized sarcoma center. Open biopsy and subsequent histopathology revealed a high grade, G3, mixed osteoblastic (20\%) and chondroblastic (80\%) osteosarcoma. Abdominal and chest CT scan showed no metastases. Therefore, in compliance with the recent guidelines, first-line therapy according to the EURAMOS-1 protocol was initiated [2]. Thus, neoadjuvant therapy for 10 weeks consisting of $120 \mathrm{mg} / \mathrm{m}^{2}$ cisplatin (P) and $75 \mathrm{mg} / \mathrm{m}^{2}$ doxorubicin (A) (weeks 1 and 6), followed by $12 \mathrm{~g} / \mathrm{m}^{2}$ high dose methotrexate (M) (weeks 4, 5, 9 and 10) were administered. Subsequently, tumor excision performing limb-sparing left-sided hemipelvectomy was performed.

Postoperative histopathology showed a complete resection (R0 resection) with a high extraosseous proportion and $20 \%$ vital tumor cells. Thus, the patient was classified as a nonresponder to neoadjuvant treatment (regression grade IV) and was subsequently assigned to adjuvant MAPIE over 28 weeks (MAP with additional ifosfamide and etoposide). Ifosfamide at the dose of $3 \mathrm{~g} / \mathrm{m}^{2}$ was given with doxorubicin over 3 days in cycles designated as $\mathrm{AI}$, and at dose of $2800 \mathrm{mg} / \mathrm{m}^{2}$ with etoposide $100 \mathrm{mg} / \mathrm{m}^{2}$ over 5 days in 

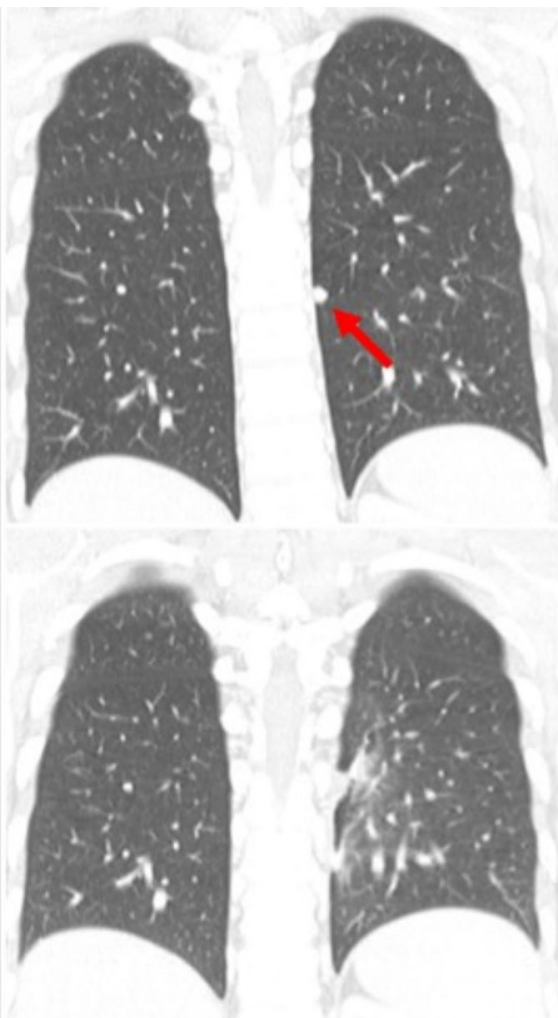

a
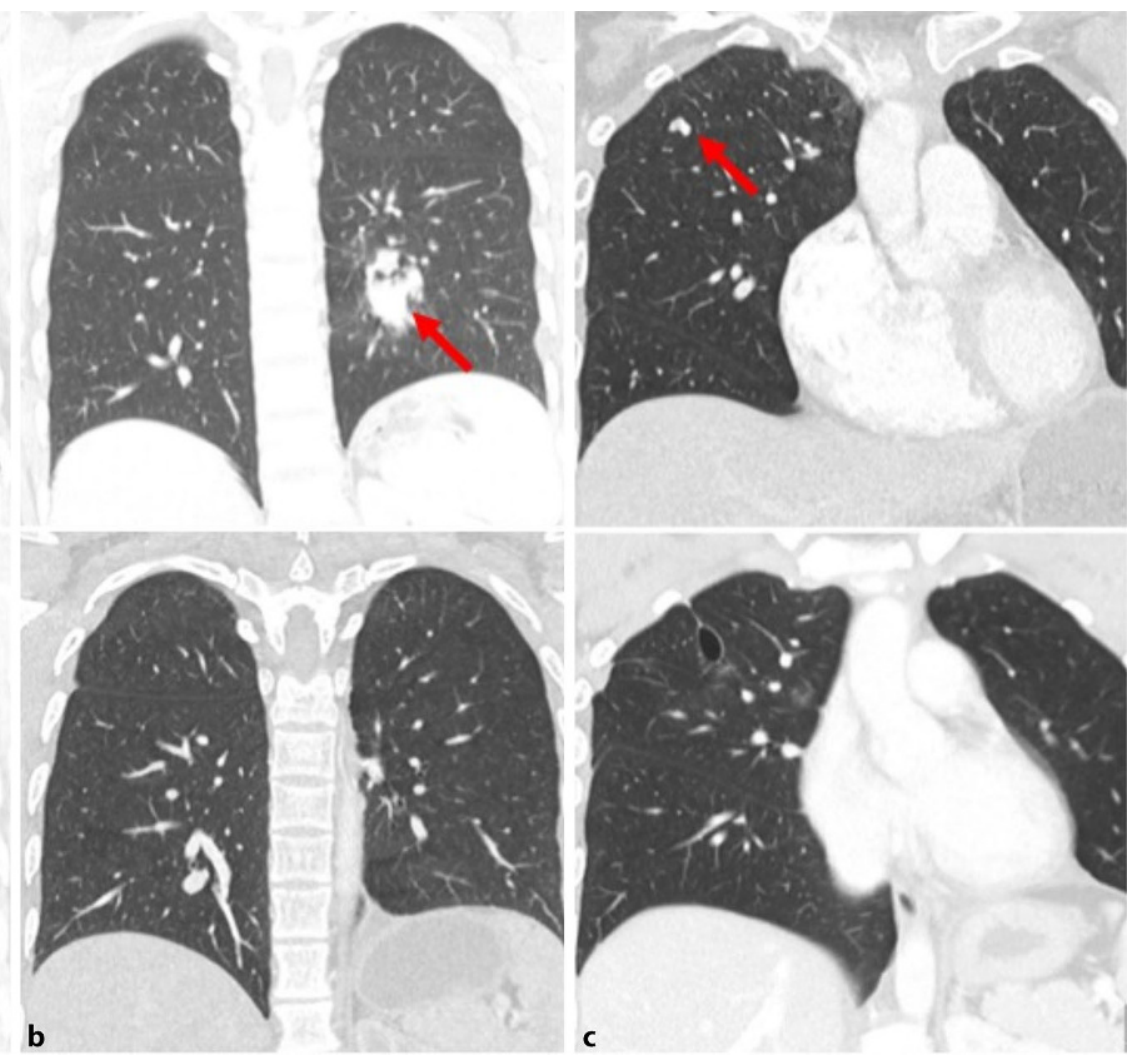

Fig. 1 CT scans of pulmonary disease progression 1-3; a October 2015, b June 2016, c August 2019. Upper segments shows the pulmonary metastasis (red arrow). Lower segments show corresponding postoperative CT scans

designated IE cycles, according to the EURAMOS-1 trial [6].

Due to neutropenic fever after IE administration (week 16), a 50\% dose reduction was necessary starting from week 19 until the end of treatment at week 39 in October 2014. The scheduled week 40 (M) was omitted due to increased liver enzymes, attributed to delayed $\mathrm{M}$ elimination in weeks 35 and 39.

Follow-up imaging via CT scan of thorax and abdomen as well as magnetic resonance imaging (MRI) of the pelvis was performed at 3 month intervals. In October 2015, a $5 \mathrm{~mm}$ lesion in the lower lobe of the left lung was identified in a chest CT scan, approximately 1 year after therapy completion. Following international guidelines, complete pulmonary metastasectomy was performed. Histopathology showed a metastasis of the chondroblastic osteosarcoma with R0 resection. Current guidelines do not recommend adjuvant treatment after complete pulmonary metastasectomy $[4,7]$. However, we chose to start adjuvant treatment in hopes of prolonging the time to potential recurrence of further pulmonary metastases and given the simple oral administration of pazopanib at home.

In June 2016, after 8 months of oral adjuvant pazopanib treatment, a CT scan showed 3 pulmonary nodules (left lower lobe: $19 \mathrm{~mm}$, lingula: $5 \mathrm{~mm}$ and right upper lobe: $4 \mathrm{~mm}$ ). Another R0 metastasectomy of all 3 pulmonary lesions was performed and experimental therapy with pembrolizumab $200 \mathrm{mg}$ every 21 days was initiated. The interval between applications of pembrolizumab was prolonged to 28 days due to pembrolizumab-induced blepharoconjunctivitis, which was successfully treated with local corticosteroids. Therapy with pembrolizumab was administered from August 2016 until July 2018, when again, a single pulmonary metastasis (right upper lobe: $4 \mathrm{~mm}$ ) was diagnosed.

A third R0 metastasectomy was performed, and an oral third-line treatment with 28-day cycles of regorafenib, another multitargeted TKI (tyrosine kinase inhibitor), at a daily dose of $40 \mathrm{mg}$, for the first 21 days of each cycle, was initiated. Apart from regorafenibinduced substitution-dependent hypothyroidism, no side effects were noted, and therapy was continued on schedule. Currently, the patient is followed up with CT scans of the thorax and abdomen every 6 months and annual MRI. Recent CT scans, 28 months after initiation of regorafenib, showed NED (no evidence of disease), regorafenib therapy is still ongoing and the patient is in excellent general condition (ECOG 0). Due to good tolerance, therapy will be continued until disease progression.

\section{Discussion and short review}

In this article, we present the case of a young patient undergoing local surgery, chemotherapy as well 
as therapy with novel agents for recurring osteosarcoma.

In 2013, according to the EURAMOS-1 trial, our patient received neoadjuvant MAP and was postoperatively randomly assigned to receive MAPIE, due to the classification as a nonresponder to neoadjuvant treatment (regression grade IV). However, in 2016, published data reported increased toxicity without survival improvement of MAPIE compared to MAP alone [6]. Therefore, current guidelines recommend local surgery as well as neoadjuvant and adjuvant treatment with only MAP in all young patients with initially localized disease [2].

Recurrence rates in bone sarcomas with complete response to initial treatment have been reported to be $20-40 \%$ [8]. In case of relapse with isolated lung metastasis, complete surgical resection, if possible, is the treatment of choice, even in consecutive relapses and is often accompanied by chemotherapy [9]. A possible chemotherapeutic option in relapsed osteosarcoma is high-dose ifosfamide with an overall response rate (ORR) of approximately $20-30 \%$ [10]. Besides ifosfamide, the use of gemcitabine and docetaxel as well as cyclophosphamide and etoposide in relapsed or refractory osteosarcoma is feasible [11, 12]. If surgery is not possible, stereotactic radiotherapy, cryotherapy or radiofrequency ablation are alternative treatment options. Evidence even suggests an effect of radiofrequency ablation and stereotactic radiotherapy as first-line treatments of resectable lung metastasis [12-14]. Only limited data exist in adjuvant therapy after pulmonary metastasectomy. Predina et al., for example, reported no differences in disease-free survival after pulmonary metastasectomy for soft tissue sarcoma with adjuvant chemotherapy versus no chemotherapy ( $p=0.88$ ) [15]. According to guidelines, watch and wait strategy is the treatment of choice in relapsed osteosarcoma patients with lung metastasis after R0 resection [7]. In this particularly young patient, however, a maximum therapy approach was chosen in hopes of prolonging disease-free survival.

Because our patient had already received first-line ifosfamide in the MAPIE arm, and did not wish any further intravenous chemotherapy treatment, other adjuvant therapeutic options had to be offered to her.

Trials investigating the efficacy of different novel treatment options, such as TKIs in osteosarcomas are scarce [16-18]. Studies on sorafenib, for example, showed an ORR between 5 and 9\% in two trials in advanced bone sarcoma patients [19, 20]. Data on the use of pazopanib, a second generation TKI, in osteosarcoma are mostly limited to small case series, demonstrating median overall survivals between 3 and 11 months in the metastatic setting $[17,18,21]$. Since R0 metastasectomy was achieved and our patient was already heavily pretreated with chemotherapy, application of off-label pazopanib was established. Therapy was well tolerated, but tumor recurrence in the lung occurred 6 months after initi- ation of therapy, which is nevertheless in the range of tumor control reported previously $[17,18,21]$.

In addition to TKIs, checkpoint inhibitors (CPI) have gained much attention in the last decade due to their widespread use in various oncologic diseases $[22,23]$. Therefore, after failure of pazopanib and second pulmonary R0 resection, experimental off-label treatment with pembrolizumab was initiated. A total of 28 cycles of pembrolizumab were administered until a third pulmonary relapse was diagnosed. During treatment with pembrolizumab, the patient showed NED over a period of 23 months with manageable side effects. However, phase II studies on the use of pembrolizumab in advanced unresectable or metastatic osteosarcoma published later showed mostly negative results with low or no antitumor effect [24-26]. In view of this, it is difficult to assess whether the course in our patient was a natural progression or if pembrolizumab had some sort of delaying effect. However, the fact that prior progression had occurred 17 months earlier makes it tempting to speculate that in the presence of minimal disease burden, pembrolizumab might still have been beneficial in our patient.

Following the third relapse and metastasectomy, our patient is currently in remission, 28 months after initiation of fourth-line regorafenib, which she tolerated well. As with our patient, previous phase 2 placebo-controlled trials demonstrate an efficacy and safety benefit of regorafenib in pretreated osteosarcoma patients. Regorafenib showed ORR between 8 and $13.6 \%$ in two phase II trials of advanced disease $[16,27]$.

\section{Conclusion}

This case demonstrated the importance of complete pulmonary metastasectomy in patients with relapsed osteosarcoma. Even after consecutive relapses, longterm survival can be achieved by complete metastasectomy, followed by adjuvant treatment with a TKI or CPI, without the necessity of further line chemotherapy administration. Therapy with TKI is feasible with manageable side effects. CPI treatment showed promising results in our patient; however due to previous conflicting data, further studies have to be established. Large phase III trials are necessary to confirm the status of adjuvant therapy after pulmonary metastasectomy.

\section{Take Home Message}

In relapsed osteosarcoma with lung metastasis, longterm survival can be achieved by $\mathrm{R} 0$ resection. In some patients adjuvant therapy with TKI is feasible with manageable side effects. 
Funding Open access funding provided by Medical University of Vienna.

Conflict of interest O. Steinbrecher, T. Brodowicz and W. Lamm declare that they have no competing interests.

Open Access This article is licensed under a Creative Commons Attribution 4.0 International License, which permits use, sharing, adaptation, distribution and reproduction in any medium or format, as long as you give appropriate credit to the original author(s) and the source, provide a link to the Creative Commons licence, and indicate if changes were made. The images or other third party material in this article are included in the article's Creative Commons licence, unless indicated otherwise in a credit line to the material. If material is not included in the article's Creative Commons licence and your intended use is not permitted by statutory regulation or exceeds the permitted use, you will need to obtain permission directly from the copyright holder. To view a copy of this licence, visit http://creativecommons.org/licenses/by/4.0/.

\section{References}

1. Stiller CA, Trama A, Serraino D, Rossi S, Navarro C, Chirlaque MD, et al. Descriptive epidemiology of sarcomas in Europe: report from the RARECARE project. Eur J Cancer. 2013;49(3):684-95.

2. Casali PG, Bielack S, Abecassis N, Aro HT, Bauer S, Biagini R, et al. Bone sarcomas: ESMO-PaedCan-EURACAN clinical practice guidelines for diagnosis, treatment and follow-up. Ann Oncol. 2018;29(4):iv79-iv95.

3. Smeland S, Bielack SS, Whelan J, Bernstein M, Hogendoorn P, Krailo MD, et al. Survival and prognosis with osteosarcoma: outcomes in more than 2000 patients in the EURAMOS-1 (European and American osteosarcoma study) cohort. Eur J Cancer. 2019;109:36-50.

4. Kempf-Bielack B, Bielack SS, Jürgens H, Branscheid D, Berdel WE, Exner GU, et al. Osteosarcoma relapse after combined modality therapy: an analysis of unselected patients in the cooperative osteosarcoma study group (COSS). JClin Oncol. 2005;23(3):559-68.

5. Briccoli A, Rocca M, Salone M, Guzzardella GA, Balladelli A, Bacci G. High grade osteosarcoma of the extremities metastatic to the lung: long-term results in 323 patients treated combining surgery and chemotherapy, 1985-2005. Surg Oncol. 2010;19(4):193-9.

6. Marina NM, Smeland S, Bielack SS, Bernstein M, Jovic G, Krailo MD, et al. Comparison of MAPIE versus MAP in patients with a poor response to preoperative chemotherapy for newly diagnosed high-grade osteosarcoma (EURAMOS1): an open-label, international, randomised controlled trial. Lancet Oncol. 2016;17(10):1396-408.

7. Casali PG, Abecassis N, Aro HT, Bauer S, Biagini R, BielackS, et al. Soft tissue and visceral sarcomas: ESMO-EURACAN clinical practice guidelines for diagnosis, treatment and follow-up. Ann Oncol. 2018;29(4):iv268-iv9.

8. García Franco CE, Torre W, Tamura A, Guillén-Grima F, SanJulian M, Martin-Algarra S, et al. Long-term results after resection for bone sarcoma pulmonary metastases. Eur J Cardiothorac Surg. 2010;37(5):1205-8.

9. BriccoliA, Rocca M, Salone M, BacciG, FerrariS, BalladelliA, et al. Resection of recurrent pulmonary metastases in patients with osteosarcoma. Cancer. 2005;104(8):1721-5.

10. Palmerini E, Setola E, Grignani G, D’Ambrosio L, Comandone A, Righi A, et al. High dose ifosfamide in relapsed and unresectable high-grade osteosarcoma patients: a retrospective series. Cells. 2020;9(11):2389.

11. Palmerini E, Jones RL, Marchesi E, Paioli A, Cesari M, Longhi A, et al. Gemcitabine and docetaxel in relapsed and unresectable high-grade osteosarcoma and spindle cell sarcoma of bone. BMCCancer. 2016;16:280.

12. Yu W, Liu Z, Tang L, Lin F, Yao Y, Shen Z. Efficacy and safety of stereotactic radiosurgery for pulmonary metastases from osteosarcoma: experience in 73 patients. Sci Rep. 2017;7(1):17480.

13. de Baere T, Tselikas L, Gravel G, Hakime A, Deschamps F, Honoré $\mathrm{C}$, et al. Interventional radiology: role in the treatment of sarcomas. Eur JCancer. 2018;94:148-55.

14. Saumet L, Deschamps F, Marec-Berard P, Gaspar N, Corradini N, Petit P, et al. Radiofrequency ablation of metastases from osteosarcoma in patients under 25 years: the SCFE experience. Pediatr HematolOncol. 2015;32(1):41-9.

15. PredinaJD, PucMM, BergeyMR, SonnadSS, KucharczukJC, Staddon A, et al. Improved survival after pulmonary metastasectomy for soft tissue sarcoma. J Thorac Oncol. 2011;6(5):913-9.

16. Duffaud F, Mir O, Boudou-Rouquette P, Piperno-Neumann S, Penel N, Bompas E, et al. Efficacy and safety of regorafenibinadultpatients with metastatic osteosarcoma: a non-comparative, randomised, double-blind, placebocontrolled, phase 2 study. Lancet Oncol. 2019;20(1):120-33.

17. Safwat A, Boysen A, Lücke A, Rossen P. Pazopanib in metastatic osteosarcoma: significant clinical response in three consecutive patients. Acta Oncol. 2014;53(10):1451-4.

18. Longhi A, PaioliA, Palmerini E, Cesari M, Abate ME, SetolaE, et al. Pazopanib in relapsed osteosarcoma patients: report on 15 cases. Acta Oncol. 2019;58(1):124-8.

19. Grignani G, Palmerini E, Ferraresi V, D'Ambrosio L, Bertulli R, Asaftei SD, et al. Sorafenib and everolimus for patients with unresectable high-grade osteosarcoma progressing after standard treatment: a non-randomised phase 2 clinical trial. Lancet Oncol. 2015;16(1):98-107.

20. Grignani G, Palmerini E, Dileo P, Asaftei SD, D’Ambrosio L, Pignochino Y, et al. A phase II trial of sorafenib in relapsed and unresectable high-grade osteosarcoma after failure of standard multimodal therapy: an Italian sarcoma group study. Ann Oncol. 2012;23(2):508-16.

21. Aggerholm-Pedersen N, Rossen P, Rose H, Safwat A. Pazopanib in the treatment of bone sarcomas: clinical experience. Transl Oncol. 2020;13(2):295-9.

22. Steininger J, Gellrich FF, Schulz A, Westphal D, Beissert S, Meier F. Systemic therapy of metastatic melanoma: on the road to cure. Cancers (Basel). 2021;13(6):1430. https:// doi. org/10.3390/cancers 13061430.

23. HerbstRS, Giaccone G, de MarinisF, ReinmuthN, VergnenegreA, Barrios $\mathrm{CH}$, etal. Atezolizumab for first-line treatment of PD-L1-selected patients with NSCLC. N Engl J Med. 2020;383(14):1328-39.

24. Boye K, Longhi A, Guren T, Lorenz S, Næss S, Pierini M, et al. Pembrolizumab in advanced osteosarcoma: results of a single-arm, open-label, phase 2 trial. Cancer Immunol Immunother. 2021; https://doi.org/10.1007/s00262-02102876-w.

25. Tawbi HA, Burgess M, BolejackV, Van Tine BA, SchuetzeSM, $\mathrm{Hu}$ J, et al. Pembrolizumab in advanced soft-tissue sarcoma and bone sarcoma (SARC028): a multicentre, twocohort, single-arm, open-label, phase 2 trial. Lancet Oncol. 2017;18(11):1493-501.

26. Le Cesne A, Marec-Berard P, Blay JY, Gaspar N, Bertucci F, Penel N, et al. Programmed cell death 1 (PD-1) targeting 


\section{short review}

in patients with advanced osteosarcomas: results from the PEMBROSARCstudy. EurJCancer. 2019;119:151-7.

27. Davis LE, Bolejack V, Ryan CW, Ganjoo KN, Loggers ET,

Chawla S, et al. Randomized double-blind phase II study of regorafenib in patients with metastatic osteosarcoma. JClin Oncol. 2019;37(16):1424-31.

Publisher's Note Springer Nature remains neutral with regard to jurisdictional claims in published maps and institutional affiliations.

- For latest news from international oncology congresses see: http://www.springermedizin.at/ memo-inoncology 